\title{
Efficient Dynamic Dictionary Matching with DAWGs and AC-automata
}

\author{
Diptarama Hendrian ${ }^{1}$, Shunsuke Inenaga ${ }^{2}$, Ryo Yoshinaka ${ }^{1}$, and \\ Ayumi Shinohara ${ }^{1}$ \\ ${ }^{1}$ Graduate School of Information Sciences, Tohoku University, Sendai, Japan, \\ \{diptarama@shino., ry@, ayumi@\}ecei.tohoku.ac.jp \\ ${ }^{2}$ Department of Informatics, Kyushu University, Fukuoka, Japan, \\ inenaga@inf .kyushu-u.ac.jp
}

\begin{abstract}
The dictionary matching is a task to find all occurrences of pattern strings in a set $D$ (called a dictionary) on a text string $T$. The Aho-Corasick-automaton (AC-automaton) which is built on $D$ is a fundamental data structure which enables us to solve the dictionary matching problem in $O(d \log \sigma)$ preprocessing time and $O(n \log \sigma+o c c)$ matching time, where $d$ is the total length of the patterns in the dictionary $D, n$ is the length of the text, $\sigma$ is the alphabet size, and occ is the total number of occurrences of all the patterns in the text. The dynamic dictionary matching is a variant where patterns may dynamically be inserted into and deleted from the dictionary $D$. This problem is called semi-dynamic dictionary matching if only insertions are allowed. In this paper, we propose two efficient algorithms that can solve both problems with some modifications. For a pattern of length $m$, our first algorithm supports insertions in $O(m \log \sigma+\log d / \log \log d)$ time and pattern matching in $O(n \log \sigma+o c c)$ for the semi-dynamic setting. This algorithm also supports both insertions and deletions in $O(\sigma m+\log d / \log \log d)$ time and pattern matching in $O(n(\log d / \log \log d+\log \sigma)+o c c(\log d / \log \log d))$ time for the dynamic dictionary matching problem by some modifications. This algorithm is based on the directed acyclic word graph (DAWG) of Blumer et al. (JACM 1987). Our second algorithm, which is based on the AC-automaton, supports insertions in $O\left(m \log \sigma+u_{f}+u_{o}\right)$ time for the semi-dynamic setting and supports both insertions and deletions in $O\left(\sigma m+u_{f}+u_{o}\right)$ time for the dynamic setting, where $u_{f}$ and $u_{o}$ respectively denote the numbers of states in which the failure function and the output function need to be updated. This algorithm performs pattern matching in $O(n \log \sigma+o c c)$ time for both settings. Our algorithm achieves optimal update time for AC-automaton based methods over constant-size alphabets, since any algorithm which explicitly maintains the AC-automaton requires $\Omega\left(m+u_{f}+u_{o}\right)$ update time.
\end{abstract}

Keywords - Dynamic dictionary matching, AC-automaton, DAWG

(c)2018. This manuscript version is made available under the CC BY-NC-ND 4.0 license 


\section{Introduction}

The pattern matching problem is, given a pattern string and a text string, to output all occurrence positions of the pattern in the text. Pattern matching is one of the most fundamental problems in string processing and has been extensively studied for decades. Efficient pattern matching algorithms are primitives for various applications such as data mining, search engines, text editors, etc.

A natural extension of the pattern matching problem is to consider a set of multiple patterns. That is, given a set $D=\left\{p_{1}, p_{2}, \ldots, p_{r}\right\}$ of patterns called a dictionary and a single text, the task is to find all occurrence positions of each pattern $p_{i} \in D$ in the text. This problem is called the dictionary matching problem [1, 4]. Aho and Corasick's algorithm (AC-algorithm) [1] and Commentz-Walter's algorithm [10] are fundamental solutions to the dictionary matching problem. For a dictionary of size $d$ over an alphabet of size $\sigma$, both of the above algorithms first preprocess the dictionary in $O(d \log \sigma)$ time. Then, given a text of length $n$, the occurrences of all patterns $p_{i} \in D$ in the text can be reported in $O(n \log \sigma+o c c)$ time by the AC-algorithm, and in $O(n d \log \sigma)$ time by the Commentz-Walter algorithm, where occ is the total number of occurrences of all the patterns in the text. Notice that $o c c \leq n d$ always holds and hence the occ term is omitted in the latter time complexity.

Meyer [21] introduced the incremental string matching problem, which is also known as the semi-dynamic dictionary matching problem, a variant of dictionary matching that allows insertion of a pattern into the dictionary. He proposed an algorithm for semidynamic dictionary matching, which updates the AC-automaton when a new pattern is inserted into the dictionary. Amir et al. [4] introduced the dynamic dictionary matching problem which allows for both insertion and deletion of patterns. Several sophisticated data structures for dynamic dictionary matching have been proposed in the literature 4, 5, 9, 18, 2, 3. . All these data structures use linear $O(d)$ (words of) space. More recently, succinct data structures for dynamic dictionary matching have been produced, where the main concern is to store the dictionary in memory space close to the information theoretical minimum [15, 17].

Remark that in all the above-mentioned approaches except Meyer's, the pattern matching time to search a text for dictionary patterns is sacrificed to some extent. Tsuda et al. 23. proposed a dynamic dictionary matching algorithm, which follows and extends Meyer's method. Whilst Tsuda et al.'s method retains $O(n \log \sigma+o c c)$ pattern matching time, still it requires $O(z \log \sigma)$ time to update the AC-automaton upon each insertion/deletion, where $z$ is the size of the AC-automaton. Note that in the worst case this can be as bad as constructing the AC-automaton from scratch, since $z$ can be as large as the dictionary size $d$. Ishizaki and Toyama [19] introduced a data structure called an expect tree which efficiently updates the dictionary for insertion of patterns and showed some experimental results, but unfortunately no theoretical analysis were provided. See Table 1 for a summary of the update times and pattern matching times for these algorithms.

Along this line, in this paper, we propose new efficient algorithms for the semidynamic and dynamic dictionary matching problems, where pattern matching can still be performed in $O(n \log \sigma+o c c)$ time.

Firstly, we show a dynamic dictionary matching algorithm which is based on Blumer 
Table 1: Comparison of the algorithms for the dynamic dictionary matching. The last four in the table are our proposed methods. Here, $n$ is the length of the text, $d$ is the total length of the dictionary of patterns, and $m$ is the length of the pattern to insert or delete. $k$ and $\epsilon$ are any constants with $k \geq 2$ and $0<\epsilon<1$, respectively. $l_{\max }$ is the length of the longest pattern in the dictionary, and $z$ is the size of the AC-automaton before updates. † indicates algorithms for semi-dynamic dictionary matching which allows only for insertion. The bounds for Chan et al.'s algorithm [8] hold for constant alphabets.

\begin{tabular}{|l|c|c|}
\hline Algorithm & Update time & Pattern matching time \\
\hline \hline Idury \& Schäffer [18] & $O\left(m\left(k d^{1 / k}+\log \sigma\right)\right)$ & $O(n(k+\log \sigma)+k \cdot o c c)$ \\
\hline Amir et al. [5] & $O\left(m\left(\frac{\log d}{\log \log d}+\log \sigma\right)\right)$ & $O\left(n\left(\frac{\log d}{\log \log d}+\log \sigma\right)+o c c \frac{\log d}{\log \log d}\right)$ \\
\hline Alstrup et al. [2, [3] & $O(m \log \sigma+\log \log d)$ & $O\left(n\left(\frac{\log d}{\log \log d}+\log \sigma\right)+o c c\right)$ \\
\hline Chan et al. [8] & $O\left(m \log ^{2} d\right)$ & $O\left((n+o c c) \log ^{2} d\right)$ \\
\hline Hon et al. [17] & $O(m \log \sigma+\log d)$ & $O(n \log d+o c c)$ \\
\hline Feigenblat et al. [16] & $O\left(\frac{1}{\epsilon} m \log d\right)$ & $O(n \log \log d \log \sigma+o c c)$ \\
\hline Meyer† [21] & $O\left(l_{\max } \cdot d \cdot \sigma\right)$ & $O(n \log \sigma+o c c)$ \\
\hline Tsuda et al. [23] & $O(z \log \sigma)$ & $O(n \log \sigma+o c c)$ \\
\hline \hline DAWG based $\dagger$ & $O(m \log \sigma)$ & $O(n \log \sigma+o c c)$ \\
\hline DAWG based & $O\left(\sigma m+\frac{\log d}{\log \log d}\right)$ & $O\left(n\left(\frac{\log d}{\log \log d}+\log \sigma\right)+o c c \frac{\log d}{\log \log d}\right)$ \\
\hline AC-automaton based $\dagger$ & $O\left(m \log \sigma+u_{f}+u_{o}\right)$ & $O(n \log \sigma+o c c)$ \\
\hline AC-automaton based & $O\left(\sigma m+u_{f}+u_{o}\right)$ & $O(n \log \sigma+o c c)$ \\
\hline
\end{tabular}

et al.'s directed acyclic word graphs (DAWGs) [6, 7]. The DAWG of a dictionary $D$ is a (partial) DFA of size $O(d)$ which recognizes the suffixes of the patterns in $D$. We show how to perform dynamic dictionary matching with DAWGs, by modifying Kucherov and Rusinowitch's algorithm which originally uses DAWGs for pattern matching with variable length don't cares [20]. The key idea is to use efficient nearest marked ancestor $(N M A)$ data structures [24, 2, 3] on the tree induced from the suffix links of the DAWG. For the semi-dynamic dictionary matching, our DAWG method achieves $O(m \log \sigma)$ update time, $O(n \log \sigma+o c c)$ pattern matching time, and uses linear $O(d)$ space, where $m$ is the length of pattern $p$ to insert. For the dynamic version of the problem, our DAWG method uses $O(\sigma m+\log d / \log \log d)$ update time, $O(n(\log d / \log \log d+\log \sigma)+$ occ $\log d / \log \log d)$ time, and $O(d)$ space. The term $\sigma m$ in the update time is indeed unavoidable for maintaining the DAWG in the dynamic setting, namely, we will also show that there is a sequence of insertion and deletion operations for patterns of length $m$ such that each insertion/deletion operation takes $\Omega(\sigma m)$ time.

Secondly, we present another algorithm for the semi-dynamic and dynamic dictionary matching problem which is based on the AC-automaton. This is closely related to our first approach, namely, the second algorithm updates the AC-automaton with the aid of the DAWG. The algorithm uses $O(d)$ space, finds pattern occurrences in the text in $O(n \log \sigma+o c c)$ time, and updates the AC-automaton in $O\left(m \log \sigma+u_{f}+u_{o}\right)$ time with additional DAWG update time, $O(m \log \sigma)$ time for semi-dynamic and $O(\sigma m)$ 
time for dynamic settings, where $u_{f}$ is the number of states whose failure link needs to be updated, and $u_{o}$ is the number of states on which the value of the output function needs to be updated. Therefore, when $u_{f}$ and $u_{o}$ are sufficiently small and $\sigma$ is constant, our update operation can be faster than other approaches. Notice that $u_{o}$ is negligible unless the pattern $p$ to insert/delete is a common prefix of many other patterns; in particular $u_{o}=0$ for any prefix codes. Also, $u_{f}$ is negligible unless the prefixes of $p$ are common substrings of many other patterns. In what follows, the update time of the AC-automaton refers to the time cost to update the $\mathrm{AC}$-automaton after insertion/deletion of a pattern. We emphasize that the update time of our algorithm

is optimal for constant-size alphabets, since any algorithm which explicitly maintains the AC-automaton must use at least $\Omega\left(m+u_{f}+u_{o}\right)$ time to update the automaton. Finally, we give tight upper and lower bounds on $u_{f}$ and $u_{o}$ in the worst case.

A preliminary version of this work appeared in [14].

\section{Preliminaries}

Let $\Sigma$ denote an alphabet of size $\sigma$. An element of $\Sigma^{*}$ is called a string. For a string $w$, the length of $w$ is denoted by $|w|$. The empty string, denoted by $\varepsilon$, is the string of length 0 . For a string $w=x y z$, strings $x, y$, and $z$ are called prefix, substring, and suffix of $w$, respectively. For a string $w$, let $\operatorname{Substr}(w)$ denote the set of all substrings of $w$, and for a set $W=\left\{w_{1}, w_{2}, \ldots, w_{r}\right\}$ of strings, let $\operatorname{Substr}(W)=\bigcup_{i=1}^{r} \operatorname{Substr}\left(w_{i}\right)$. Similarly, let $\operatorname{Pref}(W)$ be the set of all prefixes of strings in $W$. For a string $w, w[i]$ denotes the $i$-th symbol of $w$ and $w[i: j]$ denotes the substring of $w$ that begins at position $i$ and ends at position $j$.

Let $D=\left\{p_{1}, p_{2}, \ldots, p_{r}\right\}$ be a set of patterns over $\Sigma$, called a dictionary. Let $d$ be the total length of the patterns in the dictionary $D$, namely, $d=\sum_{i=1}^{r}\left|p_{i}\right|$. The Aho-Corasick Automaton [1] of $D$, denoted by $\mathrm{AC}(D)$, is a trie of all patterns in $D$, consisting of goto, failure and output functions. We often identify a state $s$ of $\operatorname{AC}(D)$ with the string obtained by concatenating all the labels found on the path from the root to the state $s$. The state transition function goto is defined so that for any two states $s, s^{\prime} \in \operatorname{Pref}(D)$ and any character $c \in \Sigma$, if $s^{\prime}=s c$ then $s^{\prime}=\operatorname{goto}(s, c)$. The failure function is defined by flink $(s)=s^{\prime}$ where $s^{\prime}$ is the longest proper suffix of $s$ such that $s^{\prime} \in \operatorname{Pref}(D)$. Finally, output $(s)$ is the set of all patterns that are suffixes of $s \cdot \operatorname{AC}(D)$ is used to find occurrences of any pattern in $D$ on a text. In this paper we omit the basic construction algorithm of $\mathrm{AC}(D)$ and how it can be used to solve the dictionary pattern matching problem (see [1, 13] for details).

For any string $x$, let

$$
\operatorname{endPos}_{D}(x)=\left\{(i, j)\left|x=p_{i}[j-|x|+1: j],\right| x|\leq j \leq| p_{i} \mid, p_{i} \in D\right\},
$$

namely, endPos ${ }_{D}(x)$ represents the set of ending positions of $x$ in patterns of $D$. For any $x, y \in \operatorname{Substr}(D)$, we define the equivalence relation $\equiv_{D}$ such that $x \equiv_{D} y$ iff endPos $_{D}(x)=\operatorname{endPos}_{D}(y)$. We denote by $[x]_{D}$ the equivalence class of $x$ with respect to $\equiv_{D}$. The directed acyclic word graph $(D A W G)$ [7, 6] of $D$, denoted by DAWG $(D)$, is an edge-labeled directed acyclic graph $(V, E)$ such that

$$
V=\left\{[x]_{D} \mid x \in \operatorname{Substr}(D)\right\}
$$




$$
E=\left\{\left([x]_{D}, c,[x c]_{D}\right) \mid x, x c \in \operatorname{Substr}(D), c \in \Sigma, x \not \equiv_{D} x c\right\} .
$$

Namely, each node ${ }^{1}$ of DAWG $(D)$ represents each equivalence class of substrings of $D$, and henceforth we will identify a DAWG node with an equivalence class of substrings. The node $[\varepsilon]_{D}$ is called the source of DAWG $(D)$. For each node $[x]_{D}$ except the source, the suffix link is defined by slink $\left([x]_{D}\right)=[y]_{D}$, where $y$ is the longest suffix of $x$ satisfying $x \not \equiv_{D} y$. For convenience, we define $\operatorname{slink}^{1}\left([x]_{D}\right)=\operatorname{slink}\left([x]_{D}\right)$ and $\operatorname{slink}^{i}\left([x]_{D}\right)=\operatorname{slink}\left(\operatorname{slink}^{i-1}\left([x]_{D}\right)\right)$ for $i>1$. A node $v$ of $\operatorname{DAWG}(D)$ is called a trunk node if there is a path from the source to $v$ which spells out some prefix of a pattern in $\operatorname{Pref}(D)$, and it is called a non-trunk node otherwise. An edge $e$ from $[x]_{D}$ to $[x c]_{D}$ labeled by $c$ is a primary edge if both $x$ and $x c$ are the longest string in their equivalence classes, otherwise it is a secondary edge. It is known (c.f. [7]) that the numbers of nodes, edges, and suffix links of $\operatorname{DAWG}(D)$ are all linear in $d$. Fig. 1 shows an example of a DAWG.

By the properties of AC-automata and DAWGs, for each state $s$ in $\mathrm{AC}(D)$, there exists a unique node $v$ in $\operatorname{DAWG}(D)$ that corresponds to $s$, so that $\mathrm{AC}(D)$ can be consistently embedded into DAWG $(D)$. Because $s \in \operatorname{Pref}(D)$, the corresponding node $v$ is a trunk node and each trunk node has its corresponding state. Therefore, there exists a one-to-one mapping from the set of trunk nodes in DAWG $(D)$ to the states of $\mathrm{AC}(D)$. We denote this mapping by $s=\pi(v)$, where $v$ is a DAWG trunk node and $s$ is the corresponding AC-automaton state. We denote $v=\pi^{-1}(s)$ iff $s=\pi(v)$. Fig. 3 (a) and (b) show the AC-automaton and DAWG of $D=\{a b b a, a c a, c b b\}$, respectively, where each number in nodes and states expresses the correspondence.

Our algorithm to follow will make a heavy use of the following lemma, which characterizes the relationship between the states of $\mathrm{AC}(D)$ and the trunk nodes of $\operatorname{DAWG}(D)$.

Lemma 1. Let $s$ and $s^{\prime}$ be any states in $\mathrm{AC}(D)$, and let $v=\pi^{-1}(s)$ and $v^{\prime}=\pi^{-1}\left(s^{\prime}\right)$ be corresponding trunk nodes in $\operatorname{DAWG}(D)$. Then, $s^{\prime}=$ flink $(s)$ iff there exists an integer $k \geq 1$ such that $v^{\prime}=\operatorname{slink}^{k}(v)$, and when $k \geq 2$, $\operatorname{slink}^{i}(v)$ is a non-trunk node for all $1 \leq i<k$.

Proof. ( $\Longrightarrow$ ) Suppose $s^{\prime}=$ flink $(s)$. Then, by definition, $s^{\prime}$ is a proper suffix of $s$. Hence there exists an integer $k \geq 1 \operatorname{such}$ that $v^{\prime}=\operatorname{slink}^{k}(v)$. Also, by definition, $k$ is the smallest such that $\operatorname{slink}^{k}(v) \in \operatorname{Pref}(D)$. Hence, when $k \geq 2$, $\operatorname{sink}^{i}(v)$ is a non-trunk node for all $1 \leq i<k$.

$(\Longleftarrow)$ Suppose there exists an integer $k \geq 1$ such that $v^{\prime}=\operatorname{slink}^{k}(v)$. When $k=1$, clearly $s^{\prime}=f \operatorname{link}(s)$. When $k \geq 2$ and $\operatorname{slink}^{i}(v)$ is a non-trunk node for all $1 \leq i<k$, then $k$ is the smallest integer such that $v^{\prime}=\operatorname{slink}^{k}(v)$ is a trunk node. Hence $s^{\prime}=f \operatorname{link}(s)$.

\section{Maintenance of Inverse Suffix Links of DAWG}

Meyer [21] and Tsuda et al. 223] used the inverse of the failure function to update the AC-automaton. Although the inverse failure function can be stored in a total of $O(d)$ space, it is not trivial whether one can efficiently access and/or update the inverse

\footnotetext{
${ }^{1}$ To avoid confusion, we refer to a vertex in DAWGs as a node, and a vertex in AC-automata as a state in this paper.
} 


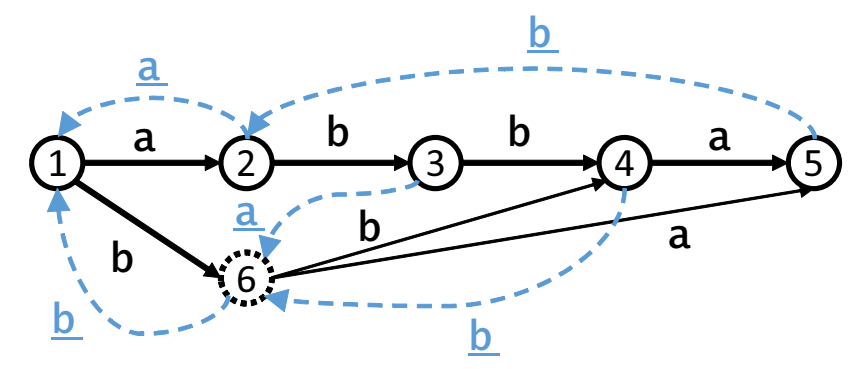

Figure 1: DAWG(\{abba $\})$. Solid-line circles show trunk nodes and the dashed-line circle shows a non-trunk node. Thick solid lines, thin solid lines, and dashed lines show primary edges, secondary edges and suffix links, respectively.

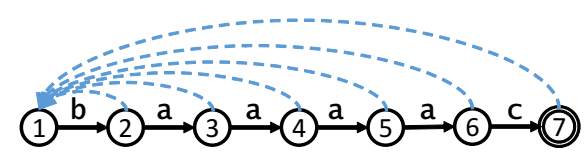

(a)

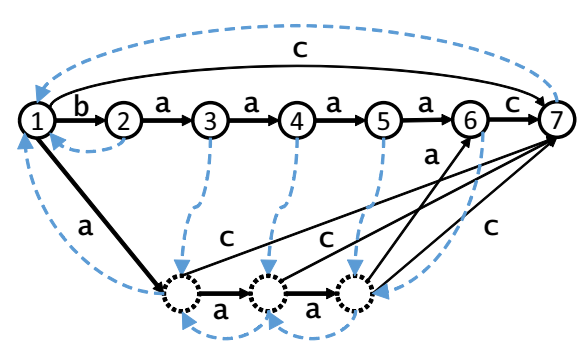

(b)

Figure 2: (a)AC $(\{$ baaaac $\})$ and (b) DAWG $(\{$ baaaac $\})$.

failure function, because the number of inverse failure links of each state may change dynamically and can be as large as the number of states in the AC-automaton. For instance, let us consider $\mathrm{AC}(D)$ for $D=\{$ baaaac $\}$ over $\Sigma=\{\mathrm{a}, \mathrm{b}, \mathrm{c}\}$ in Fig. 2 (a). Its root is pointed by 6 failure links. When a new pattern c is inserted to $D$, then the above algorithms first create a new state $s$, a new transition from the root to $s$, and a failure link from $s$ to the root. The real difficulty arises when they try to find which suffix links should be updated to point at $s$; they must follow all the 6 inverse failure links from the root and get 6 states numbered $2,3, \ldots, 7$, and check whether there is an edge labeled $c$ from each of them, although only one state 7 should be updated. Ishizaki and Toyama [19] introduced an auxiliary tree structure called an expect tree to reduce the number of the candidates and showed some experimental results, but no theoretical analysis is provided. Unfortunately, their algorithm behaves the same for the above example. Therefore, maintaining the inverse failure links to update the AC-automaton might be inefficient.

In order to overcome this difficulty, we pay our attention to the suffix links of $\operatorname{DAWG}(D)$, instead of the failure links of $\mathrm{AC}(D)$. It is known (see, e.g. [6, 7, 13, 12]) that the inverse suffix links of all nodes in $\operatorname{DAWG}(D)$ form the suffix tree of the reversed patterns in $D$, so that for any node $v$ in $\operatorname{DAWG}(D)$, each suffix link pointing at $v$ is labeled by a distinct symbol which is the first symbol of the edge label in the suffix tree. Formally, the label of a suffix link is defined as follows. Let $x y$ be the longest string in $[x y]_{D}, y$ be the longest string in $[y]_{D}$, and $\operatorname{sink}\left([x y]_{D}\right)=[y]_{D}$, the label of this suffix link is $x[|x|]$. In Fig. 1, the label of each suffix link is showed by an underlined symbol. 


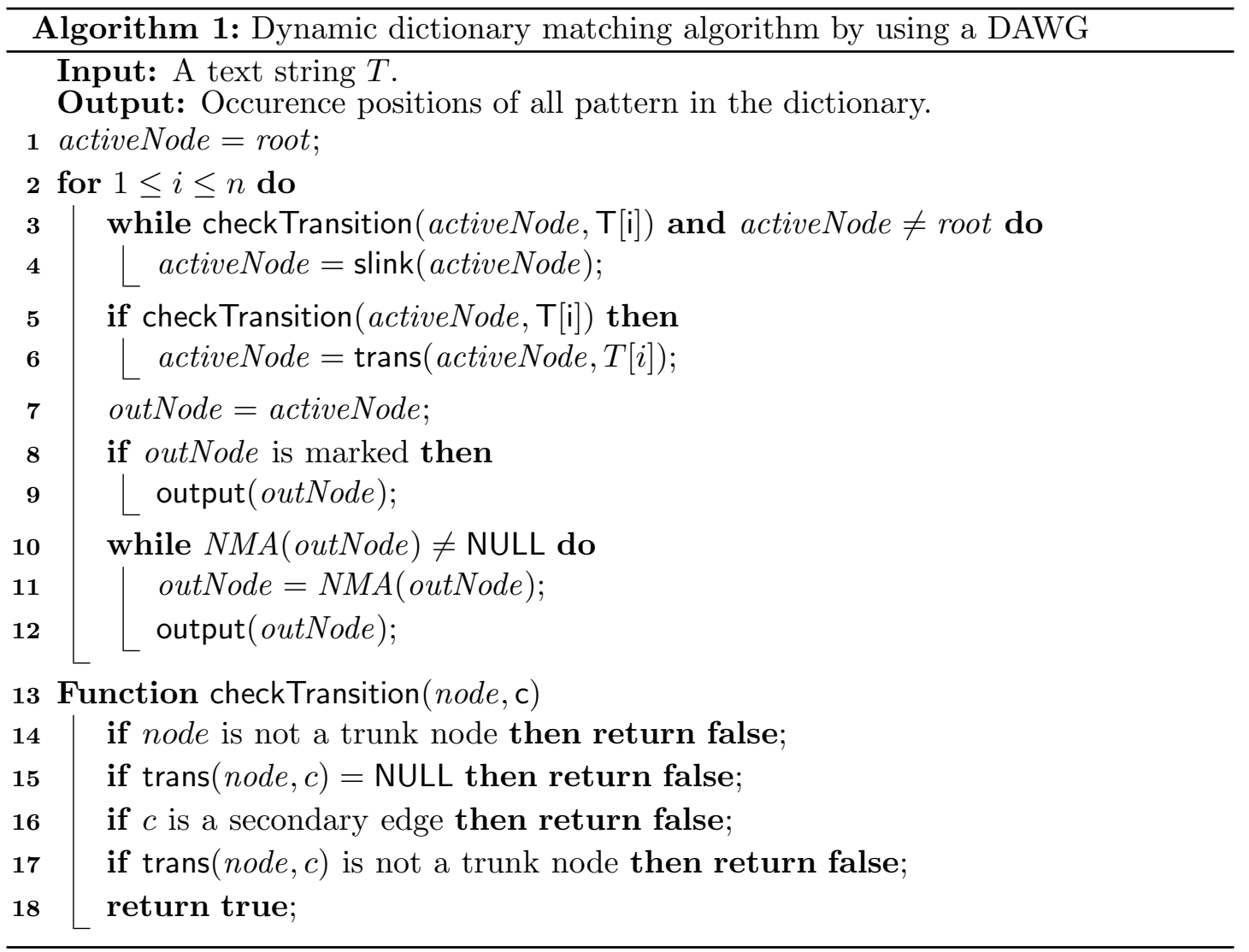

Therefore, the number of suffix links that point at $v$ is at most $\sigma$, and the inverse suffix links can be accessed and updated in $O(\log \sigma)$ time using $O(d)$ total space. This means that when a pattern of length $m$ is inserted to or deleted from the dictionary, the inverse suffix links can be maintained in $O(m \log \sigma)$ time.

It is known that DAWGs can be used for solving the pattern matching problem with a single pattern [11. However, it is not trivial to maintain the output function efficiently for dynamic and multiple patterns, as is pointed out by Kucherov and Rusinowitch [20]. In the next section, we shall show our algorithm which efficiently maintains the output function on the DAWG.

\section{Dynamic Dictionary Matching by using DAWG}

In this section, we will describe how to perform dynamic dictionary matching with the DAWG. This algorithm is a simple modification of Kucherov and Rusinowitch's algorithm [20] for matching multiple strings with variable length don't-care symbols.

First we will discuss the time complexity to update the DAWG in the semi-dynamic and dynamic settings. As it was shown in [7] the DAWG can be updated in $O(m \log \sigma)$ amortized time for an insertion of a pattern of length $m$ in the semi-dynamic setting. For the dynamic setting, Kucherov and Rusinowitch [20] gave an algorithm which deletes a pattern from the dictionary, and claimed that the update time for deletion and insertion 
is the same as in the semi-dynamic setting. However, in what follows we show that this is not true when the alphabet size is super-constant. Namely, the number of edges to be constructed when we split a DAWG node can be amortized constant by the total length of the input strings in the semi-dynamic setting, but this amortization argument does not hold in the dynamic setting. That is, we obtain the following lower bound for updating the DAWG in the dynamic setting.

Lemma 2. In the dynamic setting where both insertion and deletion of patterns are supported, there exists a family of patterns such that $\Omega(\sigma m)$ time is needed when updating the DAWG for insertion and deletion of each pattern.

Proof. To show an $\Omega(\sigma m)$ lower bound, consider a pattern $p=(\mathrm{ba})^{\frac{m}{2}}$ and an initial dictionary $D=\left\{(\mathrm{ab})^{i} \mathrm{a}^{j} c \mid 1 \leq i \leq \frac{m}{2}, j \in\{0,1\}, c \in \Sigma \backslash\{\mathrm{a}, \mathrm{b}\}\right\}$ of size $d=\Theta\left(\sigma m^{2}\right)$. We insert $p$ to the dictionary and update $\operatorname{DAWG}(D)$ into $\operatorname{DAWG}(D \cup\{p\})$. In this case we need to split a node each time we read a symbol from $p$, and construct $\sigma-2$ edges labeled by $c \in \Sigma \backslash\{\mathrm{a}, \mathrm{b}\}$ from the new node. Hence, we need to create $\Omega(\sigma m)$ edges when we update the DAWG. Moreover, the same computation time $\Omega(\sigma m)$ is required when we delete the same pattern $p$ from $D \cup\{p\}$ and update the DAWG.

If we repeat this operation more than $m$ times by inserting and deleting $p$, we cannot amortize the update cost by the size of the dictionary. Therefore, we need $\Omega(\sigma m)$ operations to update the DAWG when inserting or deleting a pattern.

The above lower bound is tight, namely, below we will show a matching upper bound for updating the DAWG in the dynamic setting.

Lemma 3. In the dynamic setting where both insertion and deletion of patterns are supported, the DAWG can be updated in $O(\sigma m)$ time for insertion and deletion of patterns.

Proof. To show the upper bound, we will evaluate the number of edges and suffix links that are traversed and/or created during the update.

Let us first consider the insertion operation. Let $p$ be a pattern of length $m$ to be inserted to the dictionary. Suppose that the prefix $p[1: i-1]$ of $p$ has already been inserted to the DAWG for $1 \leq i \leq m$. Let $v$ be the DAWG node that represents $p[1: i-1]$. There are three cases for the next pattern character $p[i]$ :

(1) There is a primary out-going edge of $v$ labeled with $p[i]$. In this case no new edge or node is created, and it takes $O(\log \sigma)$ time to traverse this primary edge.

(2) There is no out-going edge of $v$ labeled with $p[i]$. In this case, a new sink node and a new edge from $v$ to this new sink labeled with $p[i]$ are created. Then, the algorithm follows a chain of suffix links from $v$ and insert new edges leading to the new sink labeled with $p[i]$, until finding the first node which has an out-going edge labeled with $p[i]$.

(3) There is a secondary out-going edge of $v$ labeled with $p[i]$. Let $u$ be the node that is reachable from $v$ via the edge labeled with $p[i]$. This node $u$ gets split into two nodes $u$ and $u^{\prime}$, and at most $\sigma$ out-going edges of the original node $u$ are copied to $u^{\prime}$. 
It is clear that Case (1) takes $O(\log \sigma)$ time per character. At most $i$ new edges can be introduced in Case (2), but it follows from [6] that the total number of suffix links that are traversed is $O(m)$ for all $m$ characters of $p$. Hence, Case (2) takes $O(\log \sigma)$ amortized time per character. It is clear that Case (3) takes $O(\sigma)$ time. Overall, a pattern of length $m$ can be inserted to the DAWG in $O(\sigma m)$ total time.

The deletion operation can also be performed in $O(\sigma m)$ time, since the deletion can be done in the same complexity as insertion by reversing the insertion procedure (see also Kucherov and Rusinowitch's result [20]).

Next, we will describe how to find the occurrences of the patterns in the text by using the DAWG. We will use a nearest marked ancestor (NMA) data structure on the inverse suffix link tree. In the NMA problem on a rooted tree, each node of the tree is either marked or unmarked. The NMA query returns the nearest marked ancestor of a given query node $v$ in the tree, or returns NULL if $v$ has no marked ancestor. The semidynamic NMA problem allows for marking operation only, while the dynamic NMA problem allows for both marking and unmarking operations. New leaves can be added to the tree in both of the problems, and existing leaves can be removed in the dynamic problem. There is a semi-dynamic NMA data structure [24] which allows for NMA queries, marking unmarked nodes, and inserting new leaves in amortized $O(1)$ time each. For the dynamic NMA problem, there is a data structure which permits NMA queries and both marking and unmarking operations in worst-case $O(\log t / \log \log t)$ time, and inserting new leaves in amortized $O(1)$ time, where $t$ is the size of the tree [2, 3]. Both of the data structures use $O(t)$ space and $O(t)$ preprocessing time.

In our dictionary pattern matching algorithm using the DAWG, we mark each node $v$ of the inverse suffix link tree iff $v$ is a DAWG node that represents a pattern in the dictionary ${ }^{2}$ Now, for a given node $w$ in the DAWG, we can find all patterns in the dictionary that are suffixes of $w$ by performing NMA queries from $w$ on the inverse suffix link tree as follows. If $w$ itself is marked, then we output it. Then, we perform NMA queries in the inverse suffix link tree from $w$, until we find a node that has no marked ancestor. This allows us to skip all unmarked nodes in the path from $w$ to the node, and we output all marked nodes found by NMA queries in this path.

Algorithm 1 shows a pseudo-code of our algorithm for dynamic dictionary matching by using the DAWG. The algorithm only uses the trunk nodes and primary edges to perform pattern matching. Therefore, when the algorithm reads a character $c$ from the text, it checks whether or not there is a primary edge which is labeled with $c$ and leads to a trunk node by using a function checkTransition(node, $\mathrm{c}$ ). If there is no such node, then the algorithm follows a chain of suffix links until it reaches a trunk node, and then performs the same procedure as above. Thus, the suffix links of the DAWG replaces the failure links of the corresponding AC-automaton. The correctness is immediately justified by Lemma 1. As soon as the algorithm finds a primary edge which is labeled with $c$ and leads to a trunk node, it checks whether there is an occurrence of any pattern in the dictionary by using NMA queries from this destination trunk node, as

\footnotetext{
${ }^{2}$ Kucherov and Rusinowitch 20] used Sleator and Tarjan's link-cut tree data structure 22$]$ to maintain a dynamic forest induced from the inverse suffix link tree. Our important observation here is that essentially the same operations and queries in this application can be more efficiently supported with NMA data structures.
} 
described previously. This procedure is used as a substitute for the output function of the AC-automaton.

Consider inserting a new pattern $p$ to the dictionary. If $v$ is the DAWG node which represents $p$, then $v$ is newly marked in the inverse suffix link tree, and $v$ is the only node that gets marked in this stage. Hence, exactly one unmarked node gets marked per inserted pattern. For the same reasoning, exactly one marked node gets unmarked per deleted pattern. To delete an existing pattern $p$ from the dictionary and hence from the DAWG, we can use Kucherov and Rusinowitch's algorithm [20] which takes $O(\sigma m)$ time due to Lemmas 2 and 3 , where $m$ is the length of $p$.

Overall, we obtain the following.

Theorem 1. In the semi-dynamic setting where only insertion of patterns is supported, the DAWG-based algorithm supports insertion of patterns in $O(m \log \sigma)$ time and pattern matching in $O(n \log \sigma+o c c)$ time.

In the dynamic setting where both insertion and deletion of patterns are supported, the DAWG-based algorithm supports insertion/deletion in $O(\sigma m+\log d / \log \log d)$ time and pattern matching in $O(n(\log d / \log \log d+\log \sigma)+$ occ $\log d / \log \log d)$ time. The size of both data structures is $O(d)$.

Proof. The update times and space requirements of both of the semi-dynamic and dynamic versions should be clear from Lemmas 2, 3 and the above arguments.

For pattern matching, we need to perform at least one NMA query each time a character from a text is scanned, and need to perform an NMA query each time an occurrence of a pattern is found. Hence, it takes $O(n \log \sigma+o c c)$ time for the semi-dynamic setting and $O(n(\log d / \log \log d+\log \sigma)+o c c \log d / \log \log d)$ time for the dynamic setting.

\section{AC-Automaton Update Algorithm}

In this section we will describe how to perform dynamic dictionary matching by using the AC-automaton and the DAWG for the dictionary. Our algorithm performs pattern matching in exactly the same manner as the original AC-algorithm, while updating the AC-automaton dynamically with the aid of the DAWG upon insertion/deletion of patterns. We will describe how to modify the AC-automaton by using the DAWG. Note that we can simulate the AC-automaton with the DAWG augmented with the output function. However, we will explicitly use the AC-automaton since it makes the pattern matching algorithm simpler.

\subsection{Pattern insertion algorithm}

We consider inserting a new pattern $p$ of length $m$ into the dictionary $D$, and we denote the new dictionary by $D^{\prime}=D \cup\{p\}=\left\{p_{1}, p_{2}, \ldots, p_{r}, p\right\}$. It is known that $\operatorname{DAWG}(D)$ can be constructed in $O(d \log \sigma)$ time, and can be updated to $\operatorname{DAWG}\left(D^{\prime}\right)$ online in $O(m \log \sigma)$ amortized time [7]. We update $\mathrm{AC}(D)$ to $\mathrm{AC}\left(D^{\prime}\right)$ by using DAWG $(D)$, and then update $\operatorname{DAWG}(D)$ to $\operatorname{DAWG}\left(D^{\prime}\right)$. We also add weight $(v)$ to each state $v$ of $\operatorname{AC}(D)$ that is the number of occurrences $v$ as prefix in $D$. We will use weight $(v)$ as a reference counter to determine whether $v$ should be deleted or not in the deletion algorithm later. 


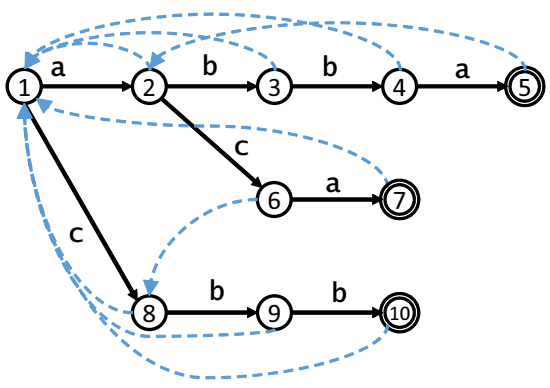

(a)

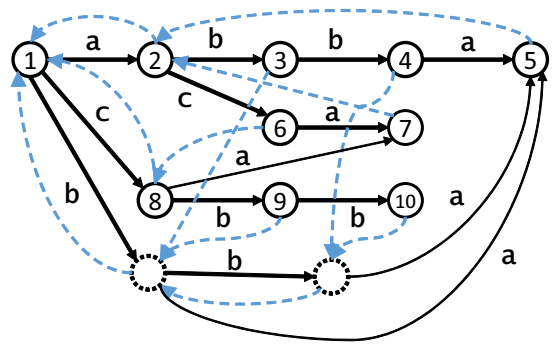

(b)

Figure 3: For a dictionary $D=\{$ abba, aca, cbb $\}$ (a) $\mathrm{AC}(D)$, and (b) DAWG $(D)$.

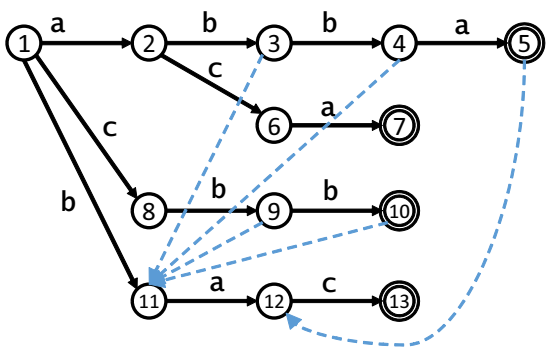

(a)

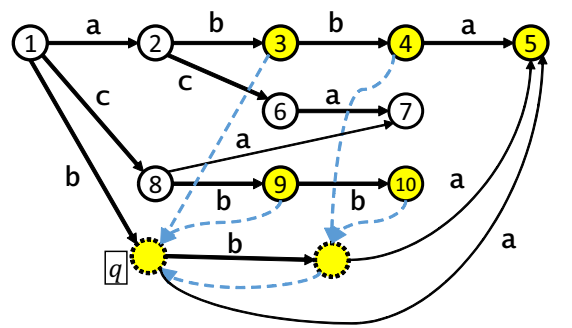

(b)

Figure 4: Illustration of updating process when inserting a pattern $p=$ bac into the dictionary $D=\{$ abba, aca, cbb $\}$. Compare them with Fig. 3. (a) The updated automaton $\operatorname{AC}\left(D^{\prime}\right)$, where the only updated failure links are shown. (b) In $\operatorname{DAWG}(D)$, the only suffix links that are used for the update are shown, and the visited nodes are colored.

The key point of our algorithm is to update the output and failure functions of $\mathrm{AC}(D)$ in linear time with respect to the number of states that should be modified. The goto function can be updated easily by adding a new transition for a new state in the same way as in the AC-automaton construction algorithm. We then update the output and failure functions efficiently by using inverse suffix links of DAWG $(D)$. Algorithm 4 updates $\mathrm{AC}(D)$ when a new pattern is inserted to $D$, and Algorithms 2 and 3 find the states on which the output and failure functions should be updated, respectively.

For any node $v$ in $\operatorname{DAWG}(D)$, let isuf $(v)=\{x \mid \operatorname{slink}(x)=v\}$ be the set of its inverse suffix links. The set isuf $(v)$ for each $v$ is stored in an ordered array $v_{a}$ as described in Section 3 . For the new pattern $p$, we can divide $p$ to $p=x y z$ and categorize the prefixes of $p$ into three categories, so that for any $i, j, k$ with $1 \leq i \leq|x|<j \leq|x|+|y|<k \leq m$;

1. $p[1: i]$ exists both in $\operatorname{AC}(D)$ and $\operatorname{DAWG}(D)$,

2. $p[1: j]$ does not exist in $\operatorname{AC}(D)$ but exists in $\operatorname{DAWG}(D)$, and

3. $p[1: k]$ exists in neither $\mathrm{AC}(D)$ nor $\operatorname{DAWG}(D)$.

To update both output and failure functions of $\mathrm{AC}(D)$ to $\mathrm{AC}\left(D^{\prime}\right)$ we only use nodes in $\operatorname{DAWG}(D)$ that represent prefixes in the second category. Algorithm 2 follows inverse suffix links of a node representing $p$ recursively in $\operatorname{DAWG}(D)$, in order to find all the states in $\mathrm{AC}(D)$ on which the output function needs to be updated. On the other hand, 


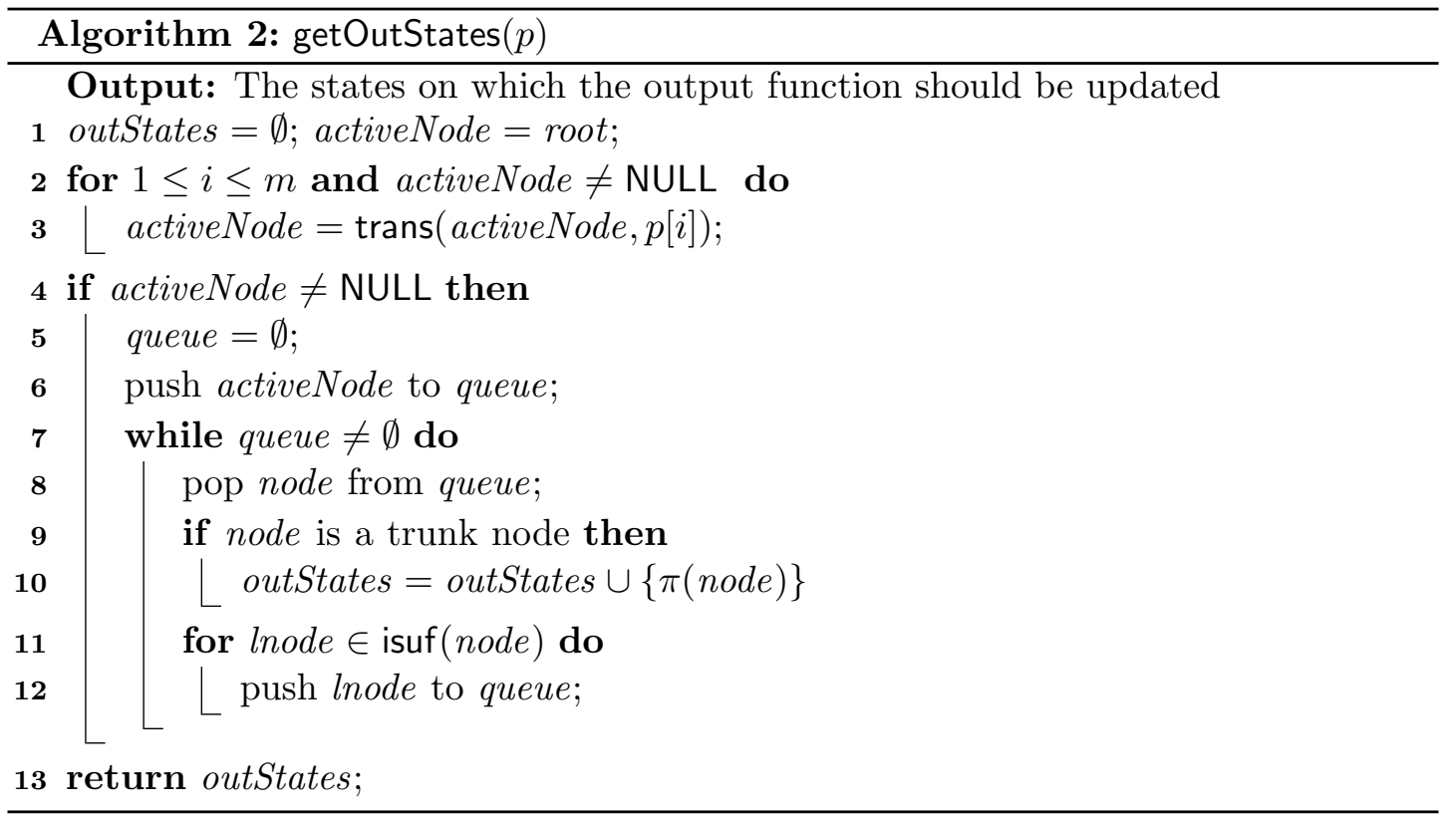

Algorithm 3 follows inverse suffix links of nodes that represent $p[i: j]$ for $|x|<j \leq$ $|x|+|y|$ (category 2) recursively, until it reaches a trunk node $u$, and then saves the state $s=\pi(u)$ that corresponds to the trunk node to update its failure link later.

Fig. 4 illustrates an example, where we insert a pattern $p=$ bac into the dictionary $D=\{$ abba, aca, cbb $\}$. First, we create new states 11, 12, and 13 . The string $\mathrm{b}$ is represented by node $q$ in $\operatorname{DAWG}(D)$, and by the new state 11 in $\operatorname{AC}\left(D^{\prime}\right)$, thus there is at least one state whose failure link should be updated to point at the state 11 . We will explain how to find these states below. Similarly, we know that at least one failure link should be updated to point at the state 12, because the string ba represented by the state 12 in $\mathrm{AC}\left(D^{\prime}\right)$ is also represented by node 5 in $\operatorname{DAWG}(D)$. However, the string bac, which is represented by the new state 13, is not represented in DAWG $(D)$, thus we know that there is no state whose failure link should be updated to state 13 . As a result, we have the set $\{11,12\}$ of states. (Lines 25 in Algorithm 3 )

We now explain how to find states whose failure links should be updated. We begin by the deepest state in $\{11,12\}$, that is, state 12 . We search the states from node 5 in $\operatorname{DAWG}(D)$, which represents the same string ba as state 12 in $\mathrm{AC}\left(D^{\prime}\right)$. When searching from node 5 , we do not search further because node 5 is a trunk node. Therefore, we update the failure link of state 5 to state 12 . Next, to find states whose failure links should be updated to state 11 , we search the states from node $q$ in $\operatorname{DAWG}(D)$, which represents the same string $\mathrm{b}$ as state 11 in $\mathrm{AC}\left(D^{\prime}\right)$. By following the inverse suffix links recursively from node $q$ until reaching a trunk node, we get the set $\{3,4,9,10\}$ of trunk nodes (see Fig. 4 (b)). Therefore, we update the failure links of states 3, 4, 9, and 10 to state 11 . (Lines 6 18) 


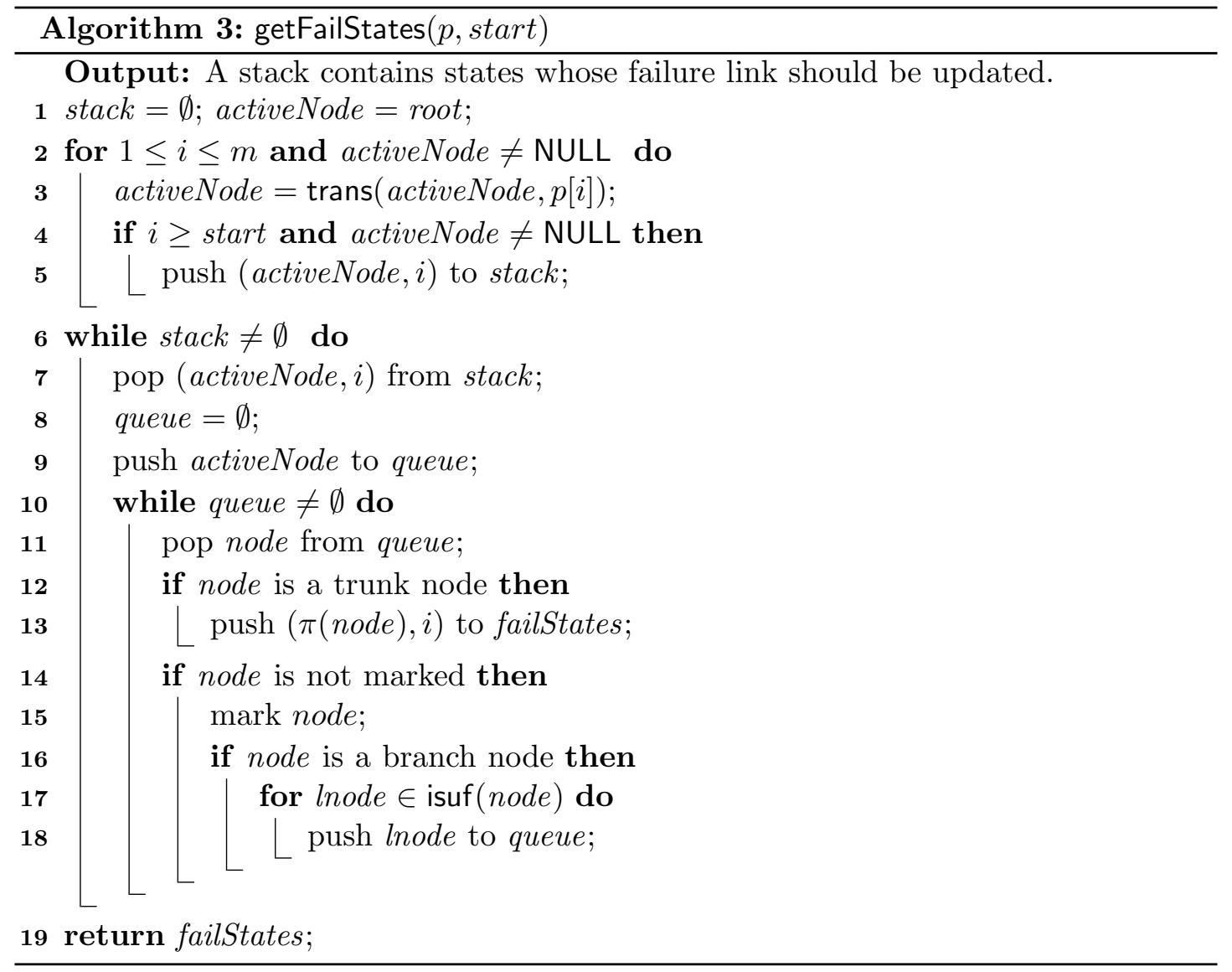

\subsection{Pattern deletion algorithm}

We consider deleting a pattern $p_{i}$ of length $m$ from the dictionary $D$, and we denote the new dictionary by $D^{\prime}=D \backslash\left\{p_{i}\right\}=\left\{p_{1}, \ldots, p_{i-1}, p_{i+1}, \ldots, p_{r}\right\}$. Similarly to insertion, we can delete a pattern from $\operatorname{DAWG}(D)$ in $O(m \log \sigma)$ time [20]. We update $\operatorname{AC}(D)$ to $\operatorname{AC}\left(D^{\prime}\right)$ by using $\operatorname{DAWG}(D)$, and then update $\operatorname{DAWG}(D)$ to $\operatorname{DAWG}\left(D^{\prime}\right)$. The proposed deletion algorithm also updates the output and failure functions of $\mathrm{AC}(D)$ in linear time with respect to the number of states that should be modified.

Algorithm 5 shows the proposed deletion algorithm. First, the algorithm finds which states should be deleted. The algorithm finds the states by decreasing the weight of states which represent prefixes of $p$. The algorithm will delete the states whose weight becomes zero, which means those states do not represent any prefix of any pattern in $D^{\prime}$.

After the algorithm has found the states which should be deleted, it will update the states whose failure links should be updated. A state should be updated if the failure link of the state is pointing at one of the nodes that will be deleted. Such states can be found by traversing reverse failure links of the states. From Lemma 1 we can use inverse suffix links of the DAWG instead of inverse failure links of the AC-automaton to find the states. The algorithm uses getFailStates $(p, s t a r t)$ in Algorithm 3 to find the states and update them from the states of which the suffix links point to shallower states. 


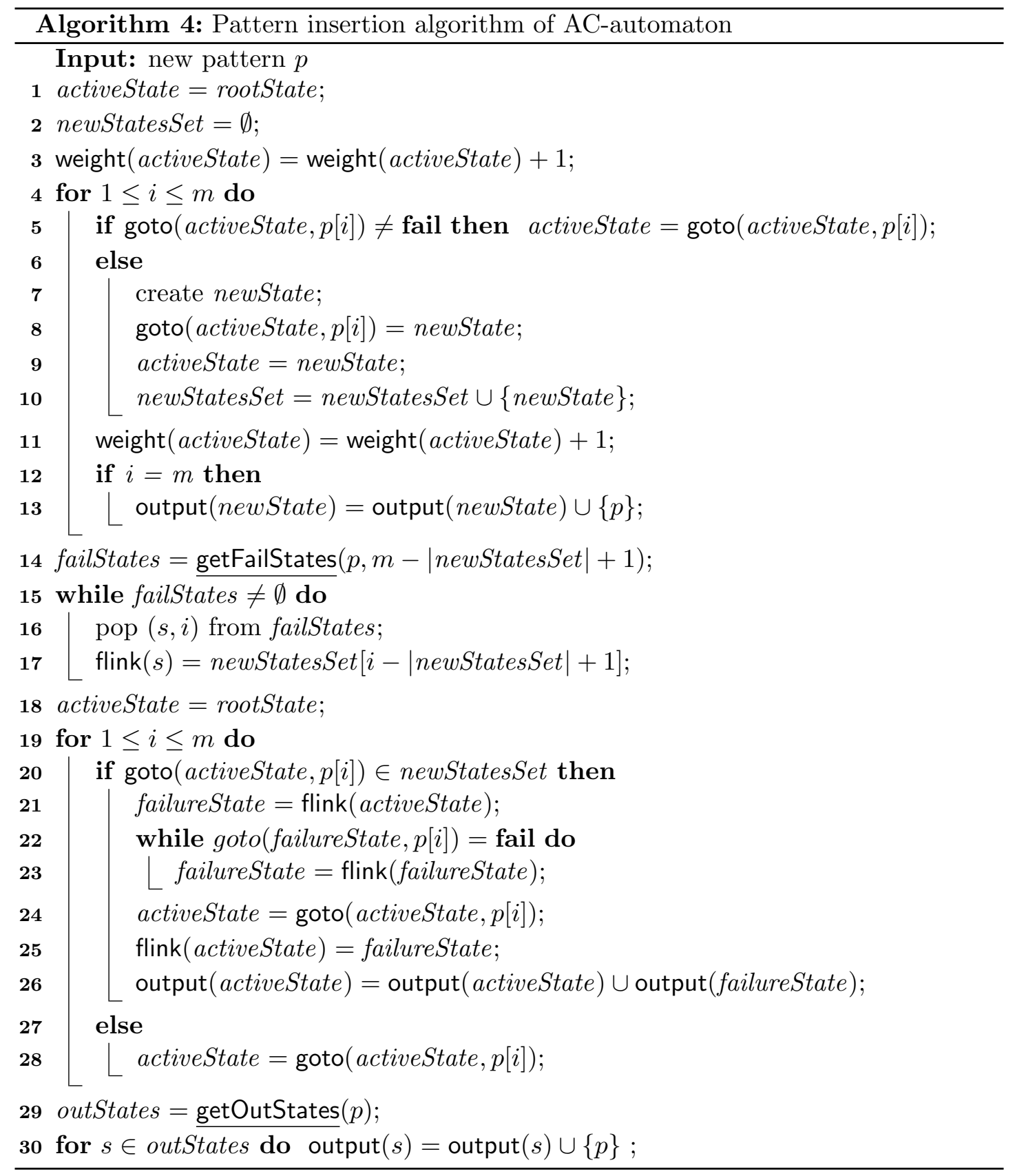




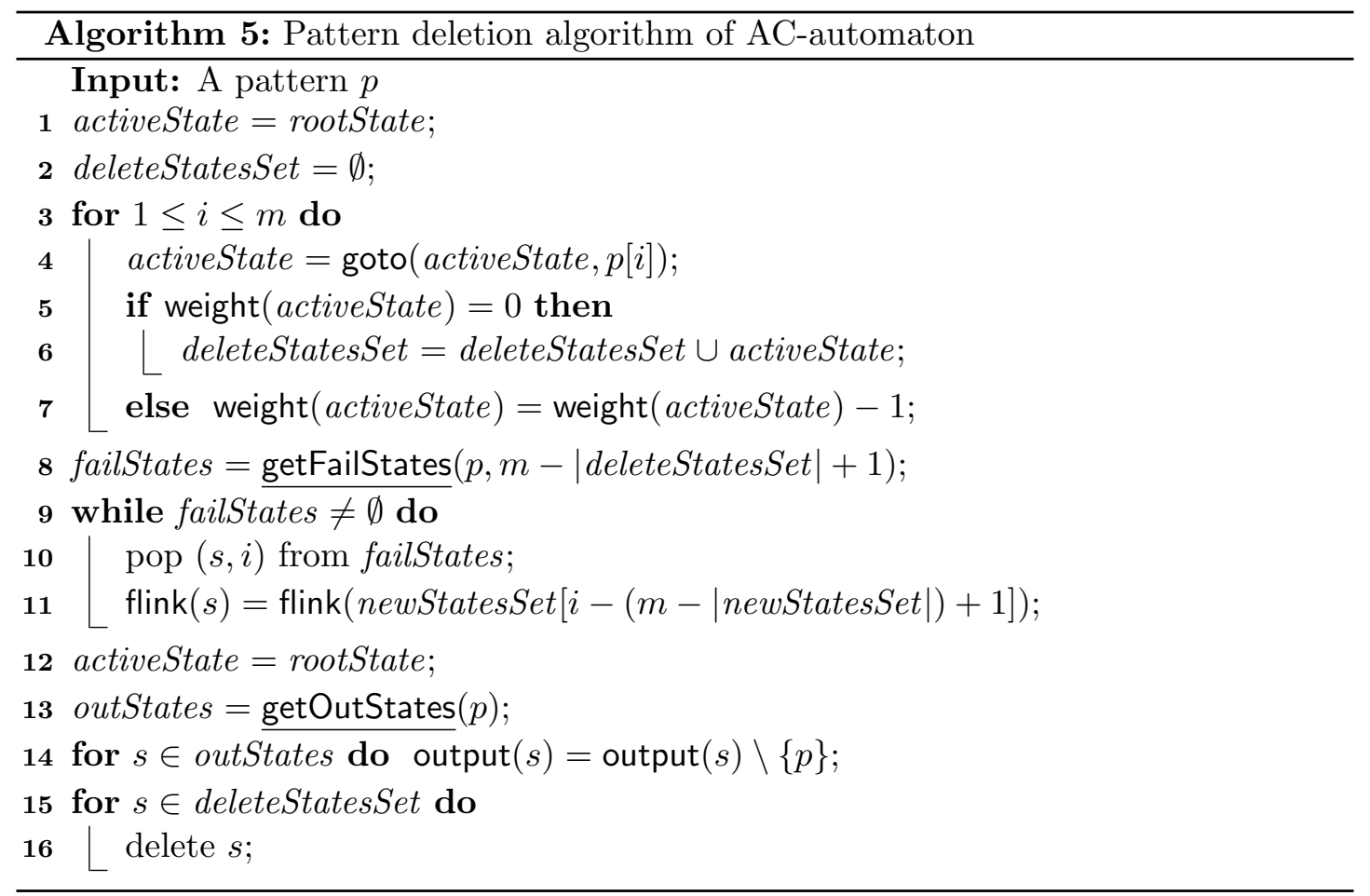

Next, the algorithm will update the output function of the AC-automaton. The output function of a state should be updated if and only if $p$ is a suffix of the string that is represented by the state. The algorithm uses getOutStates $(p)$ in Algorithm 2 to find the states whose output function should be updated. Last, the algorithm will delete the respective states.

\subsection{Correctness of the algorithms}

We now show the correctness of Algorithms 2 and 3 .

Lemma 4. Algorithm 2 correctly returns the set of states on which output functions should be updated.

Proof. When a new pattern $p$ is inserted to a dictionary $D$, we have to update the output function of every state $s$ in $\operatorname{AC}(D)$ such that $p$ is a suffix of the string $s$. If there is no node in $\operatorname{DAWG}(D)$ representing $p$, we know that no such a string $s$ exists in $D$. Otherwise, let $s_{p}$ be a new state created in $\mathrm{AC}\left(D^{\prime}\right)$ to represent the pattern $p$. The output function of some state $s$ should be updated if and only if $s_{p}$ is reachable from $s$ via a chain of failure links. From Lemma 1, for nodes $u=\pi^{-1}(s)$ and $v_{p}=[p]_{D}$, we have $v_{p}=\operatorname{slink}^{i}(u)$ for some $i$. Therefore, $s=\pi(u)$ can be found by following inverse suffix links from $v_{p}$ recursively.

Lemma 5. Algorithm 3 correctly returns the set of states whose failure links should be updated. 
Proof. By arguments similar to the proof of Lemma 4, all the states that should be updated are reachable via chains of inverse suffix links from the nodes in DAWG $(D)$ that correspond to the new states in $\mathrm{AC}\left(D^{\prime}\right)$. Next, we will show that Algorithm 3 only returns the set $S$ of the states that should be updated. Let $x$ be a new state and $t=[x]_{D}$ be a node that represents the string $x$. Assume that $S$ contains a state $s$ that can be reached by following inverse failure links from $x$ recursively but should not be updated. Let $u=\pi^{-1}(s)$ and $v=\pi^{-1}($ flink $(s))$ be trunk nodes in $\operatorname{DAWG}(D)$ corresponding to $s$ and flink $(s)$, respectively. From Lemma 1, $v=\operatorname{slink}^{i}(u)$ and $t=\operatorname{slink}^{j}(v)$ for some $i$ and $j$. Since Algorithm 3, started from $t$, stops a recursive search after reaching a trunk node ( $v$ in this case), it would not find $u$. Therefore, $s=\pi(u) \notin S$.

\section{Algorithm Complexity Analysis}

We now show the time complexity of Algorithms 2 and 3 .

Lemma $6([6])$. A string $x \in \operatorname{Substr}(D)$ is the longest member of $[x]_{D}$ if and only if either $x \in \operatorname{Pref}(D)$ or $a x, b x \in \operatorname{Substr}(D)$ for some distinct $a, b \in \Sigma$.

Lemma 7. For any non-trunk node in DAWG, there exist at least two suffix links that point at it.

Proof. Let $[x]_{D}$ be any non-trunk node in $\operatorname{DAWG}(D)$ and $x \in \operatorname{Substr}(D)$ be the longest member of $[x]_{D}$. Then $x \notin \operatorname{Pref}(D)$ because $[x]_{D}$ is a non-trunk node. By Lemma 6 , there exist two distinct $a, b \in \Sigma$ such that $a x, b x \in \operatorname{Substr}(D)$. Because $x$ is the longest member of $[x]_{D}$, we have $[a x]_{D} \neq[x]_{D}$. Thus, slink $\left([a x]_{D}\right)=[x]_{D}$ because $x$ is a suffix of $a x$. Similarly, slink $\left([b x]_{D}\right)=[x]_{D}$. Because $[a x]_{D} \neq[b x]_{D}$, the non-trunk node $[x]_{D}$ is pointed by at least two suffix links.

Lemma 8. Algorithm 2 runs in $O\left(m \log \sigma+u_{o}\right)$ time, where $u_{o}$ is the number of states on which output function should be updated.

Proof. At first, Algorithm 2 finds the node $v$ representing the pattern $p$, by traversing the nodes from the root, in Lines 2 3 . It takes $O(m \log \sigma)$ time. If it failed, done. Then we analyze the running time consumed in Lines $4-12$ by counting the number $\ell$ of visited nodes in DAWG $(D)$. These nodes form a tree, rooted at $v$ and connected by inverse suffix links chains. Let $g_{n}$ (resp. $g_{t}$ ) be the number of non-trunk (resp. trunk) nodes in this tree, and let $q$ be the number of nodes (either non-trunk or trunk) that are child nodes of some non-trunk node. Because every non-trunk node has at least two child nodes by Lemma 7, we have $2 g_{n} \leq q$, and obviously $q \leq g_{n}+g_{t}$. Thus, $g_{n} \leq g_{t}$, which yields that $\ell=g_{n}+g_{t} \leq 2 g_{t}=2 \mid$ outStates $\mid=2 u_{o}$. Therefore, Algorithm 2 runs in $O\left(m \log \sigma+u_{o}\right)$ time.

Lemma 9. Algorithm 3 runs in $O\left(m \log \sigma+u_{f}\right)$ time, where $u_{f}$ is the number of states whose failure links should be updated.

Proof. At first, Algorithm 3 finds the set $V$ of nodes representing the pattern $p[1: j]$ for $1 \leq j \leq m$ such that $p[1: j]$ does not exist in $\operatorname{AC}(D)$ but does exist in $\operatorname{DAWG}(D)$, by traversing the nodes from the root, in Lines 25 . The algorithm saves the nodes in a 
stack, because the algorithm will search from the deepest node. This takes $O(m \log \sigma)$ time. Then we analyze the running time consumed in Lines $6-18$ by counting the number $\ell$ of visited nodes in DAWG $(D)$. These nodes form a forest, where each tree is rooted by some node in $V$ and connected by inverse suffix link chains, where some node in $V$ can be an inner node of a tree rooted by another in $V$. In this case, we mark the nodes that have been visited, so each node is visited at most twice. Let $g_{n}$ (resp. $g_{t}$ ) be the number of non-trunk (resp. trunk) nodes in this forest, and let $q$ be the number of nodes (either non-trunk or trunk) that are child nodes of some non-trunk node. Because every nontrunk node has at least two child nodes by Lemma 7, we have $2 g_{n} \leq q$, and obviously $q \leq g_{n}+g_{t}$. Thus, $g_{n} \leq g_{t}$, which yields that $\ell=g_{n}+g_{t} \leq 2 g_{t}=2 \mid$ failStates $\mid=2 u_{f}$.

Theorem 2. AC-automaton can be updated for each pattern in $O\left(m \log \sigma+u_{f}+u_{o}\right)$ time.

Proof. The goto, failure and output functions of newly created states can be calculated in $O(m \log \sigma)$, similarly to the original AC-automaton construction algorithm. From Lemmas 8 and 9 , the output and failure functions on existing states can be updated in $O\left(m \log \sigma+u_{o}\right)$ and $O\left(m \log \sigma+u_{f}\right)$, respectively. Therefore, AC-automaton can be updated in $O\left(m \log \sigma+u_{f}+u_{o}\right)$ time in total.

Note that any algorithm which explicitly updates the AC-automaton requires at least $\Omega\left(m+u_{f}+u_{o}\right)$ time. Hence, the bound in the above theorem is optimal except for the term $\log \sigma$ which can be ignored for constant alphabets. As was stated in Section 1 . $u_{f}$ and $u_{o}$ can be considerably small in several cases.

The remaining question is how large $u_{f}$ and $u_{o}$ can be in the worst case. The next theorem shows matching upper and lower bounds on $u_{f}$ and $u_{o}$.

Theorem 3. For any pattern of length $m, u_{f}=O(\mathrm{~km})$ and $u_{o}=O(\mathrm{~km})$, where $k$ is the number of patterns to insert to the current dictionary. Also, there exists a family of patterns for which $u_{f}=\Omega(\mathrm{km})$ and $u_{o}=\Omega(\mathrm{km})$.

Proof. In this proof, we only show bounds for $u_{f}$; however, the same bounds for $u_{o}$ can be obtained similarly.

First, we show an upper bound $u_{f}=O(\mathrm{~km})$. We begin with an empty dictionary and insert patterns to the dictionary. Let $d$ be the total length of the patterns in the dictionary after adding all patterns, and let total_ $u_{f}$ be the total number of ACautomaton states whose failure links need to be updated during the insertion of all patterns. If $k$ is the number of patterns to insert, then clearly total_ $u_{f} \leq k d$ holds. Hence, the number of failure links to update per character is total_ $u_{f} / d \leq k$. This implies that for any pattern of length $m$, the number $u_{f}$ of failure links to update is $O(k m)$.

To show a lower bound $u_{f}=\Omega(k m)$, consider an initial dictionary $D=\left\{c_{i} a^{k} \mid 1 \leq\right.$ $i \leq x\}$ of $x \geq 1$ patterns, where $k \geq 1$ and $c_{i} \neq c_{j}$ for any $1 \leq i \neq j \leq x$. For each $j=1,2, \ldots, k$ in increasing order, we insert a new pattern $a^{j}$ to the dictionary. Then, the total number total_ $u_{f}$ of failure links to update for all $a^{j}$ 's is

$$
\text { total_u } u_{f}=x k+x(k-1)+x(k-2)+\cdots+x=x k(k+1) / 2 .
$$


Let $d_{a d d}$ be the total length of patterns to insert to the initial dictionary, and $d$ the total length of the patterns after adding all patterns to the initial dictionary. Then $d=x k+d_{a d d}=x k+k(k+1) / 2$. Hence, the number of failure links to update for each character in the added patterns $a^{j}$ 's is

$$
\frac{t o t a l_{-} u_{f}}{d_{a d d}}<\frac{t o t a l_{-} u_{f}}{d}=\frac{x k(k+1)}{2 x k+k(k+1)}=\frac{x(k+1)}{2 x+k+1}=\Omega\left(\frac{x k}{x+k}\right),
$$

which becomes $\Omega(k)$ by choosing $x=\Omega(k)$. Hence, for each $1 \leq m \leq k$, when we add pattern $a^{m}$ of length $m$ to the dictionary, $u_{f}=\Omega(\mathrm{km})$ failure links need to be updated.

The arguments in the above proof consider the semi-dynamic case where only insertion of new patterns in supported. However, if we delete all patterns after they have been inserted, then exactly the same number of failure links need to be updated. Hence, the same matching upper and lower bounds hold also for the dynamic case with both insertion and deletion of patterns.

\section{Conclusions and Future Work}

We proposed two new algorithms for dynamic dictionary matching, based on DAWGs and AC-automata.

The semi-dynamic version of our first method, which uses the DAWG, supports updates (insertions of patterns) in $O(m \log \sigma)$ time and pattern matching in $O(n \log \sigma+o c c)$ time, while the dynamic version supports updates (insertions and deletions of patterns) in $O(m(\log d / \log \log d+\log \sigma))$ time and pattern matching in $O(n(\log d / \log \log d+$ $\log \sigma)+o c c \log d / \log \log d)$ time. Our second method supports updating the AC-automaton in $O\left(m \log \sigma+u_{f}+u_{o}\right)$ time with the additional DAWG update time, and pattern matching in $O(n \log \sigma+o c c)$ time. Here, $m, \sigma, n, o c c, d, u_{f}$, and $u_{o}$ respectively denote the length of the pattern to insert/delete, the alphabet size, the length of the text, the number of occurrences of patterns in the text, the total length of the patterns, the number of AC-automaton states on which the failure functions need to be updated, and the number of AC-automaton states on which the output functions need to be updated. Since $u_{f}$ and $u_{o}$ are the minimum costs to explicitly update the AC-automaton, our second method is faster than any existing dynamic dictionary matching algorithms based on AC-automata [19, 21, 23].

An intriguing open question is whether or not one can achieve $O(m \log \sigma)$ update time and $O(n \log \sigma+o c c)$ pattern matching time for dynamic dictionary matching allowing for both insertions and deletions of patterns.

\section{Acknowledgments}

The research of Diptarama, Ryo Yoshinaka, and Ayumi Shinohara is supported by Tohoku University Division for Interdisciplinary Advance Research and Education, JSPS KAKENHI Grant Numbers JP15H05706, JP24106010, and ImPACT Program of Council for Science, Technology and Innovation 
(Cabinet Office, Government of Japan). The research of Shunsuke Inenaga is in part supported by JSPS KAKENHI Grant Number $17 \mathrm{H} 01697$.

\section{References}

[1] A. V. Aho and M. J. Corasick. Efficient string matching: an aid to bibliographic search. Communications of the ACM, 18(6):333-340, 1975.

[2] S. Alstrup, T. Husfeldt, and T. Rauhe. Marked ancestor problems. In FOCS 1998, pages 534-544, 1998.

[3] S. Alstrup, T. Husfeldt, and T. Rauhe. Marked ancestor problems. Technical Report RS-98-7, BRICS, 1998.

[4] A. Amir, M. Farach, Z. Galil, R. Giancarlo, and K. Park. Dynamic dictionary matching. Journal of Computer and System Sciences, 49(2):208-222, 1994.

[5] A. Amir, M. Farach, R. M. Idury, J. A. Lapoutré, and A. A. Schäffer. Improved dynamic dictionary matching. Information and Computation, 119(2):258-282, 1995.

[6] A. Blumer, J. Blumer, D. Haussler, A. Ehrenfeucht, M.-T. Chen, and J. Seiferas. The smallest automation recognizing the subwords of a text. Theoretical Computer Science, 40:31-55, 1985.

[7] A. Blumer, J. Blumer, D. Haussler, R. McConnell, and A. Ehrenfeucht. Complete inverted files for efficient text retrieval and analysis. J. ACM, 34(3):578-595, July 1987.

[8] H. Chan, W. Hon, T. W. Lam, and K. Sadakane. Compressed indexes for dynamic text collections. ACM Trans. Algorithms, 3(2):21, 2007.

[9] H.-L. Chan, W.-K. Hon, T.-W. Lam, and K. Sadakane. Dynamic dictionary matching and compressed suffix trees. In SODA 2005, pages 13-22, 2005.

[10] B. Commentz-Walter. A string matching algorithm fast on the average. In H. A. Maurer, editor, ICALP 1979, pages 118-132, 1979.

[11] M. Crochemore. String matching with constraints. In MFCS 1988, pages 44-58, 1988.

[12] M. Crochemore and W. Rytter. Text algorithms. Oxford University Press, Inc., 1994.

[13] M. Crochemore and W. Rytter. Jewels of Stringology. World Scientific Publishing Co. Pte. Ltd., 2002.

[14] Diptarama, R. Yoshinaka, and A. Shinohara. AC-automaton update algorithm for semi-dynamic dictionary matching. In SPIRE 2016, pages 110-121, 2016.

[15] G. Feigenblat, E. Porat, and A. Shiftan. An improved query time for succinct dynamic dictionary matching. In CPM 2014, pages 120-129, 2014. 
[16] G. Feigenblat, E. Porat, and A. Shiftan. A grouping approach for succinct dynamic dictionary matching. Algorithmica, 77(1):134-150, 2017.

[17] W.-K. Hon, T.-W. Lam, R. Shah, S.-L. Tam, and J. S. Vitter. Succinct index for dynamic dictionary matching. In ISAAC 2009, pages 1034-1043, 2009.

[18] R. M. Idury and A. A. Schäffer. Dynamic dictionary matching with failure functions. Theoretical Computer Science, 131(2):295-310, 1994.

[19] F. Ishizaki and M. Toyama. An incremental update algorithm for large AhoCorasick automaton. In Proceedings of the 4 th Forum on Data Engineering and Information Management, F11-5, pages 1-6, 2012. (In Japanese).

[20] G. Kucherov and M. Rusinowitch. Matching a set of strings with variable length don't cares. Theoretical Computer Science, 178(12):129 - 154, 1997.

[21] B. Meyer. Incremental string matching. Information Processing Letters, 21(5):219$227,1985$.

[22] D. D. Sleator and R. E. Tarjan. A data structure for dynamic trees. J. Comput. Syst. Sci., 26(3):362-391, 1983.

[23] K. Tsuda, M. Fuketa, and J. Aoe. An incremental algorithm for string pattern matching machines. International journal of computer mathematics, 58:33-42, 1995.

[24] J. Westbrook. Fast incremental planarity testing. In ICALP 1992, pages 342-353, 1992. 